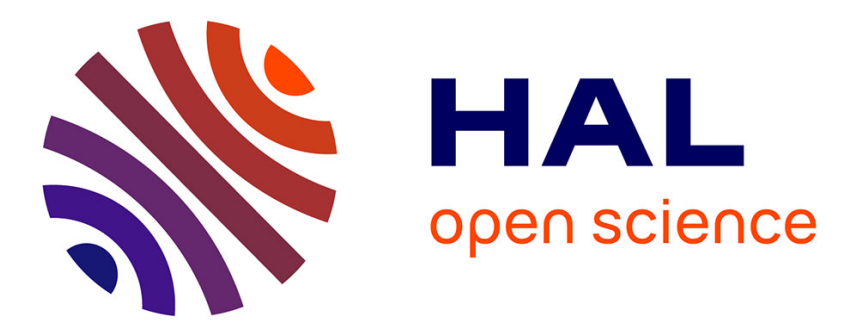

\title{
Polarization insensitive Ge-rich silicon germanium waveguides for optical interconnects on silicon
}

V Vakarin, Papichaya Chaisakul, Jacopo Frigerio, Andrea Ballabio, Joan

Manel Ramírez, Xavier Le Roux, Jean-René Coudevylle, Laurent Vivien, Giovanni Isella, Delphine Marris-Morini

\section{To cite this version:}

V Vakarin, Papichaya Chaisakul, Jacopo Frigerio, Andrea Ballabio, Joan Manel Ramírez, et al.. Polarization insensitive Ge-rich silicon germanium waveguides for optical interconnects on silicon. SPIE optics + Optoelectronics, Apr 2017, Pragues, Czech Republic. hal-01617222

\section{HAL Id: hal-01617222 \\ https://hal.science/hal-01617222}

Submitted on 16 Oct 2017

HAL is a multi-disciplinary open access archive for the deposit and dissemination of scientific research documents, whether they are published or not. The documents may come from teaching and research institutions in France or abroad, or from public or private research centers.
L'archive ouverte pluridisciplinaire HAL, est destinée au dépôt et à la diffusion de documents scientifiques de niveau recherche, publiés ou non, émanant des établissements d'enseignement et de recherche français ou étrangers, des laboratoires publics ou privés. 


\title{
Polarization insensitive Ge-rich silicon germanium waveguides for optical interconnects on silicon
}

\author{
V.Vakarin* ${ }^{\mathrm{a}}$, Papichaya Chaisakul ${ }^{\mathrm{a}}$, Jacopo Frigerio ${ }^{\mathrm{b}}$, Andrea Ballabio ${ }^{\mathrm{b}}$, Joan Manel Ramírez ${ }^{\mathrm{a}}$, \\ Xavier Le Roux ${ }^{\text {a }}$, Jean-René Coudevylle ${ }^{\text {a }}$, Laurent Vivien ${ }^{\text {a }}$, Giovanni Isella ${ }^{b}$, Delphine Marris- \\ Morini $^{\mathrm{a}}$ \\ ${ }^{a}$ Centre de Nanosciences et de Nanotechnologies,CNRS, Univ.Paris-Sud, Université Paris Saclay, \\ C2N-Orsay, 91405 cedex France; \\ ${ }^{\mathrm{b}}$ L-NESS, Dipartimento di Fisica del Politecnico di Milano, Polo di Como, Via Anzani 42, I 22100 \\ Como, Italy;
}

\begin{abstract}
We propose germanium-rich silicon germanium waveguides as a basic building block for polarization insensitive circuitry on silicon. In this work a detailed study of SiGe waveguides geometries is performed to find optimal parameters to simultaneously obtain low polarization sensitivity and single mode operation at $\lambda=1.55 \mu \mathrm{m}$. The polarization dependence of the effective index, group index and dispersion coefficient is investigated. Optimized geometries are tolerant to fabrication errors and can be realized with the current state of the art CMOS technology. As a next step polarization insensitive multimode interference structures have been designed.
\end{abstract}

Keywords: Silicon photonics, Germanium, Waveguide, polarization insensitive, MMI

\section{INTRODUCTION}

With increasing data consumption communications, systems and data centers are pushed to their limits. New solutions have to be introduced to obtain large bandwidth, fast and low power consumption communication systems. Optical interconnects have been identified as an efficient solution. In this context, a CMOS compatible platform is of great interest to take advantage of mature and low cost silicon $(\mathrm{Si})$ technology. Germanium $(\mathrm{Ge})$ is a promising candidate for a unified photonic platform on silicon. For the past decade, Ge pseudo direct band gap properties have been used to demonstrate: laser sources ${ }^{1,2}$, efficient modulators ${ }^{3,4}$ and photodetectors ${ }^{5-8}$ with low power consumption and remarkable high speed performances. However, straightforward integration of Ge rich high quality active devices with passive circuitry is difficult due to $4 \%$ lattice mismatch between $\mathrm{Si}$ and Ge. To avoid high dislocation rate and efficiently accommodate $\mathrm{Si}$ and Ge lattice parameters an integration scheme based on a graded buffer layer can be used. Using this approach we demonstrated low loss waveguides by confining light in Ge rich SiGe layer placed on top of the graded buffer. This approach leads to the integration of Ge based active devices: germanium quantum well active region embedded in a PIN diode can be grown on Ge-rich $\mathrm{Si}_{1-\mathrm{y}} \mathrm{Ge}_{\mathrm{y}}$ virtual substrate that also acts like a guiding layer ${ }^{9}$.Moreover, we recently have demonstrated the possibility to obtain low footprint $90^{\circ}$ turns and asymmetric Mach Zehnder interferometers ${ }^{10}$. Up to now, inly TE-mode propagation has been considered, while polarization dependence is an inherent problem to integrated photonic circuits. In the following, we show that these Ge-rich $\mathrm{Si}_{1-\mathrm{x}} \mathrm{Ge}_{\mathrm{x}}$ waveguides can also be used to tackle the polarization dependence. A detailed study of waveguide geometries is performed to find optimal parameters leading to low polarization sensitivity single mode waveguides at $\lambda=1.55 \mu \mathrm{m}$. Optimized geometries are tolerant to fabrication errors as they allow more than $10 \mathrm{~nm}$ etching depth errors which is larger than typical tolerance in CMOS foundries. Based on these waveguides multimode interference structures (MMI) have been designed to operate simultaneously in TE and TM polarizations. This work paves the way to the polarization insensitive passive circuitry on bulk silicon substrate. 


\section{MATERIAL}

The vertical profile of the waveguide is reported in Fig 1a. It is composed of $2 \mu \mathrm{m}$ thick relaxed $\mathrm{Si}_{0.2} \mathrm{Ge}_{0.8}$ guiding layer on top of a $\mathrm{Si}_{1-\mathrm{x}} \mathrm{Ge}_{\mathrm{x}}$ graded buffer. The maximal Ge concentration of the $\mathrm{SiGe}$ alloy is $80 \%$ on top of the structure. This value places indirect absorption band edge below $1.55 \mu \mathrm{m}$ wavelength. Additionally, Ge concentration is linearly increasing from 0 to 0.79 in the growth direction over $11 \mu \mathrm{m}$ thickness. This linear increase results in the linear refractive index evolution from 3.477 to 4.112 at $1.55 \mu \mathrm{m}$ wavelength. The refractive index evolution along the growth direction of the epilayer is shown in Fig1.(b). Thus, with high quality low energy plasma enhanced chemical vapor deposition (LEPECVD) this epilayer (Fig. 1(a)) can be used to obtain low loss Ge-rich SiGe waveguides, while the germanium concentration of the guiding layer is high enough to be used as a virtual substrate for further growth of Ge rich layers for active components ${ }^{9}$

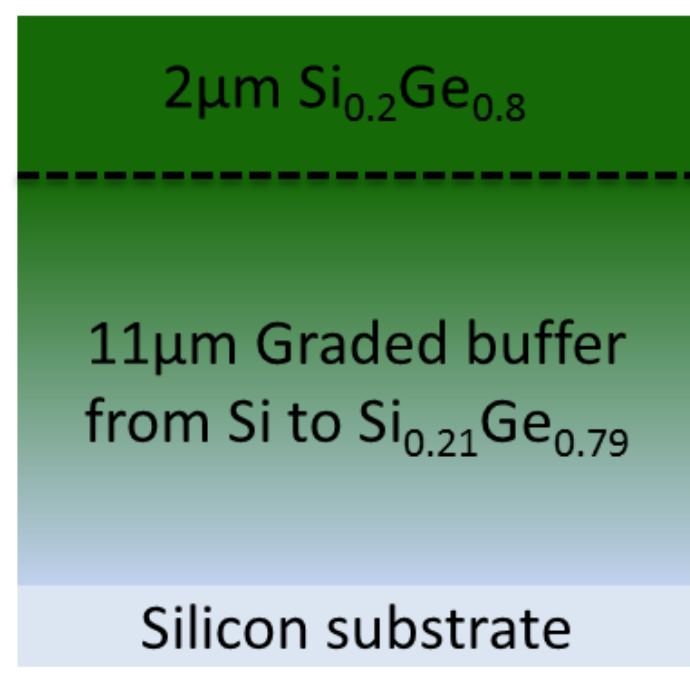

(a)

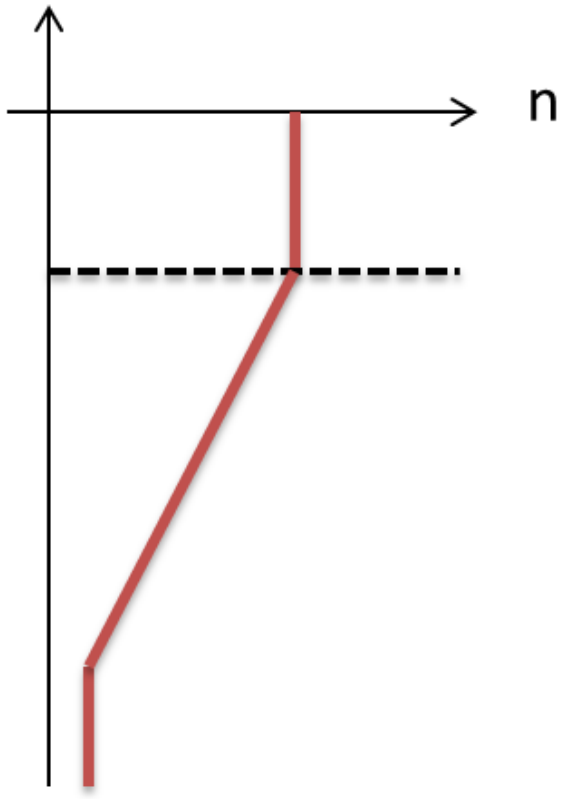

(b)

Figure 1. Ge-rich SiGe material platform: (a) epilayer grown by LEPECVD on standard silicon substrate, (b) refractive index evolution along the growth direction 


\section{WAVEGUIDE SIMULATION}

The birefringence can be defined as the difference between effective indexes between TE and TM modes or as the difference between their group indexes. Effective index difference will induce a phase difference between TE and TM modes after propagation in the waveguide, which is proportional to this effective index difference and to the waveguide length. However the waveguide geometry can be optimized to minimize this value. Additionally single mode operation is usually preferred. Hence, numerical simulations have been performed to find waveguide geometries that satisfy these requirements. Effective indexes of TE and TM modes have been calculated for etching depths ranging from $0.2 \mu \mathrm{m}$ to $2 \mu \mathrm{m}$ and widths from 1 to $3 \mu \mathrm{m}$. The effective index difference is reported in Fig. 2(a).

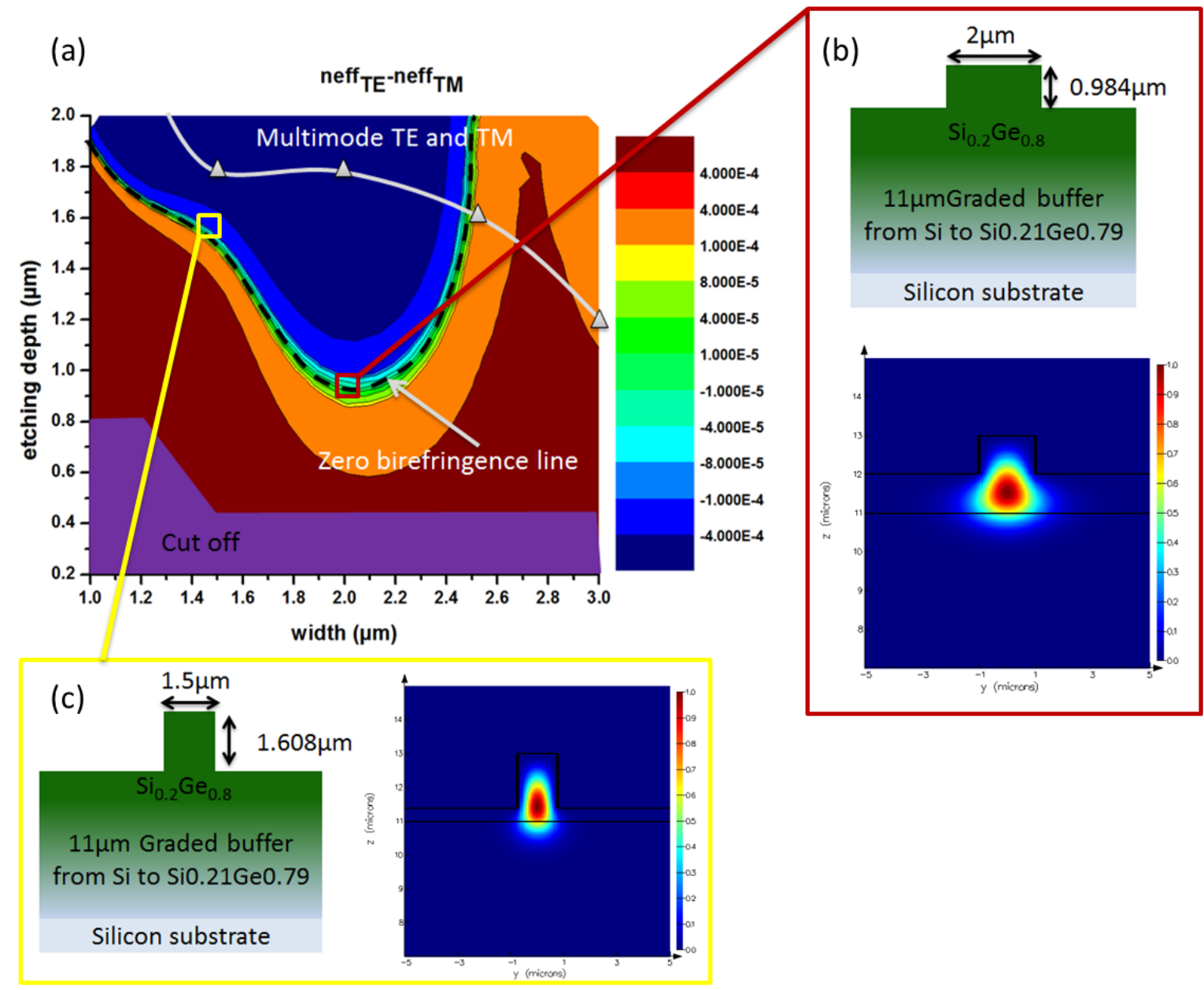

Figure 2. Waveguide effective index birefringence optimization: (a) $\Delta$ neff $=$ neff ${ }_{\mathrm{TE}}-$ neff $_{\mathrm{TM}}$ maps, (b) cross section and optical mode of optimized waveguide: configuration 1 (c) cross section and optical mode of optimized waveguide: configuration 2

Effective index birefringence can be canceled for etching depths between 0.9 and $2 \mu \mathrm{m}$ and waveguide widths between 1 and $2.5 \mu \mathrm{m}$ according to the zero birefringence line. In the same time single mode operation can be achieved with those geometries. However, most of these geometries are not viable due to the fabrication errors that can be introduced during device processing. Hence, among low birefringence geometries matching single mode propagation condition, the most stable to variations have to be selected. Consequently, two configurations have been chosen: Fig.2 (b), configuration 1 and Fig.2(c), configuration 2. These two geometries give an effective index difference between TE and TM modes as low as $1 \mathrm{E}-6$ which means that the modes will be $\pi$ shifted only after propagation over $77.5 \mathrm{~cm}$ distance. This value is far 
bigger than the typical distances used for modern chipscale interconnects and makes SiGe waveguides a particularly interesting solution for polarization dependence problem. The fabrication tolerance margins have been calculated for these waveguides and are reported below in Table 1. These margins give the typical allowed values for fabrication errors that still give $\Delta$ neff as low as $1 \mathrm{E}-4$, (corresponding to a $\pi$ shift after a propagation length of $7.75 \mathrm{~mm}$ ). As seen, the configuration 1 (waveguide width $=2 \mu \mathrm{m}$, etching depth $=0.984 \mu \mathrm{m}$ ) is more robust in terms of fabrication errors and is hence more suitable for passive circuitry implementation.

\begin{tabular}{|c|c|c|c|c|}
\hline & Widening & Narrowing & Overetching & Underetching \\
\hline $\begin{array}{l}\text { Configuration 1: } \\
\text { Width }=2 \mu \mathrm{m} \\
\text { Etching } \\
\text { depth }=0.984 \mu \mathrm{m}\end{array}$ & $90 \mathrm{~nm}$ & $50 \mathrm{~nm}$ & $10 \mathrm{~nm}$ & $10 \mathrm{~nm}$ \\
\hline $\begin{array}{l}\text { Configuration 2: } \\
\text { Width }=1.5 \mu \mathrm{m} \\
\text { Etching } \\
\text { depth }=1.608 \mu \mathrm{m}\end{array}$ & $80 \mathrm{~nm}$ & $5 \mathrm{~nm}$ & $5 \mathrm{~nm}$ & $10 \mathrm{~nm}$ \\
\hline
\end{tabular}

Table 1. Fabrication tolerance of selected geometries

For applications sensitive to group index difference such as arrayed waveguide gratings (AWG), the behavior of the group index with waveguide geometrical parameters have to be investigated in TE and TM polarizations. The group indexes were calculated as following ${ }^{11}$ : for each waveguide the effective index dependency with the wavelength has been fitted with a $5^{\text {th }}$ order polynomial function and the group indexes are deduced according to the equation (1):

$$
n g=n e f f-\lambda \frac{\partial n e f f}{\partial \lambda} .
$$

As a result, group index birefringence mappings were obtained (Fig.3)

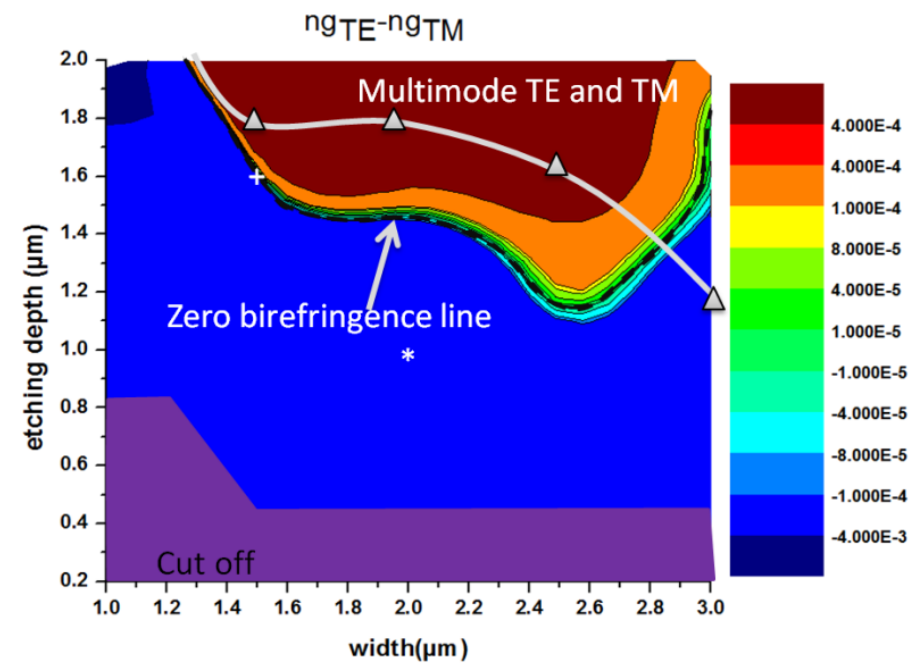

Figure 3. Influence of geometrical parameters on group index birefringence: $\Delta \mathrm{ng}=\mathrm{ng}_{\mathrm{TE}}-\mathrm{ng}_{\mathrm{TM}}$ maps, ${ }^{*}$-configuration 1 waveguide position, +-configuration 2 waveguide position

As can be seen from the mappings, configuration 1 and configuration 2 waveguides have a higher birefringence from group index point of view: $-1 \mathrm{E}-3$ and $-1 \mathrm{E}-4$ respectively. Even if using the configuration 2 waveguide a $\pi$ phase shift between TE and TM propagation modes is obtained for propagation length higher than $7 \mathrm{~mm}$, this design is not tolerant to the fabrication errors and thus makes it unusable for processing. For the configuration 1, the group birefringence is high. 
Consequently, a different geometry has to be optimized if we target low group index birefringence. For instance, $2 \mu \mathrm{m}$ waveguide have to be etched down to $1.467 \mu \mathrm{m}$ to obtain a group index birefringence lower than $1 \mathrm{E}-5$. It has to be noted that transitions are possible between different waveguide geometries in the same chip, to benefit from optimized designs for each part of the circuit.

Finally it is also interesting to investigate the dispersion coefficient. In a dispersive medium, signals with different frequencies will propagate with different velocities thus the shape of a propagating pulse will be affected. Through the dispersion coefficient one can evaluate the pulse distortion. Hence, second derivative of the effective index have been used to calculate the dispersion coefficient for TE and TM polarizations. TE dispersion coefficient and dispersion coefficient birefringence are reported in Fig.4 below:

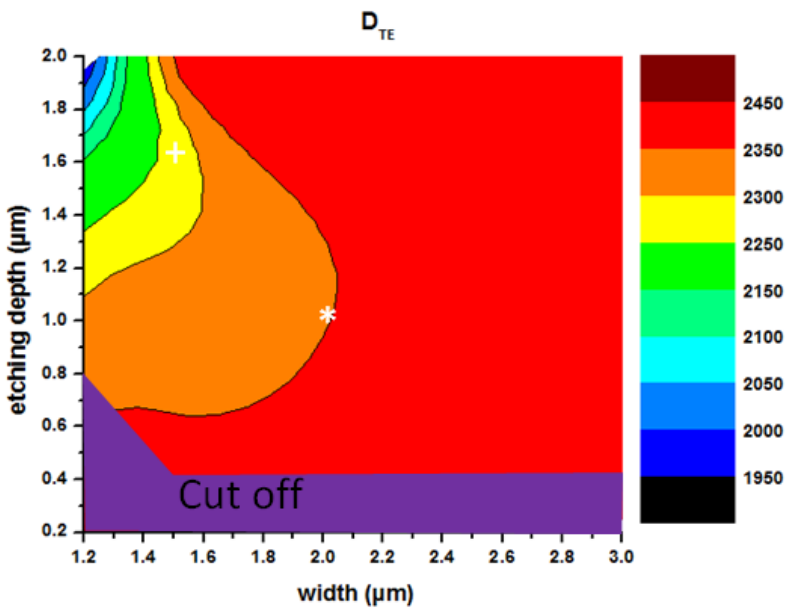

(a)

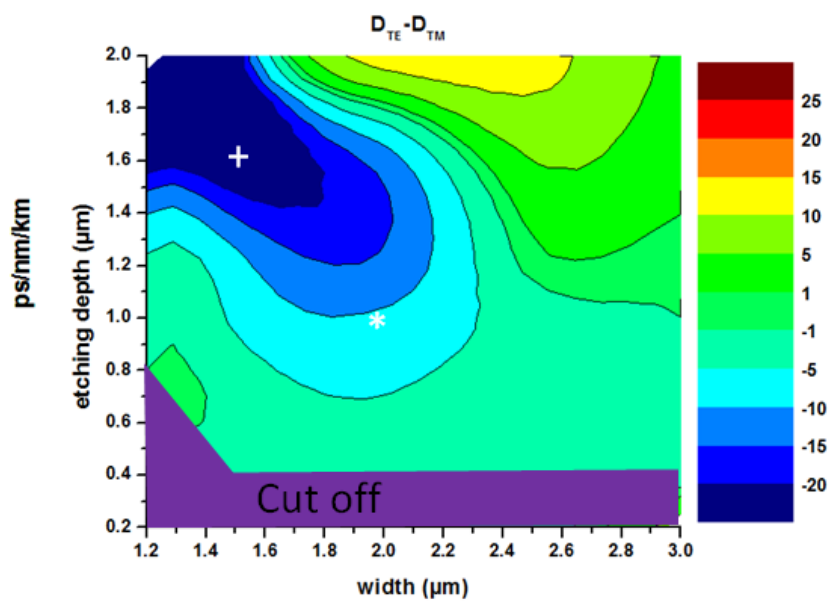

(b)

Figure 4. Influence of geometrical parameters on: TE dispersion coefficient (a), dispersion coefficient birefringence $\mathrm{D}_{\mathrm{TE}^{-}}$ $\mathrm{D}_{\mathrm{TM}}(\mathrm{b})$, *configuration 1 waveguide position, +-configuration 2 waveguide position

From dispersion coefficient calculation several interesting properties of Ge-rich SiGe waveguide arise. Ge-rich waveguides have positive dispersion coefficient: between 1950 and $2500 \mathrm{ps} / \mathrm{nm} / \mathrm{km}$ depending on geometrical configuration. The value of this coefficient is of the same order of magnitude that the absolute value of the one obtained in SOI waveguides ${ }^{12}$.Interestingly, dispersion coefficient birefringence for both configurations is quite low: $20 \mathrm{ps} / \mathrm{nm} / \mathrm{km}$ for configuration 2 and $5 \mathrm{ps} / \mathrm{nm} / \mathrm{km}$ for configuration one. Configuration 1 is preferred as it gives lower dispersion coefficient birefringence and simultaneously low effective index birefringence and fabrication robustness. Small difference between dispersion coefficients in TE and TM indicates that pulse dispersion during propagation in TE and TM polarization will be quite similar. 


\section{POLARIZATION INSENSITIVE MMI}

Based on the waveguide geometry study and to go further, a polarization insensitive 1x2 MMI has been designed based on the $1^{\text {st }}$ selected configuration $(2 \mu \mathrm{m}$ wide and $0.984 \mu \mathrm{m}$ etched waveguide). The optimized MMI design is presented in Fig.4 (a). Field intensity profiles for TE and TM polarizations are reported in Fig.4 (b) and Fig.4 (c). The device performance is quite similar for both polarizations. Indeed, $91.8 \%$ transmission is obtained in both cases. Based on this splitter, polarization insensitive Mach-Zehnder interferometer can be demonstrated in future.
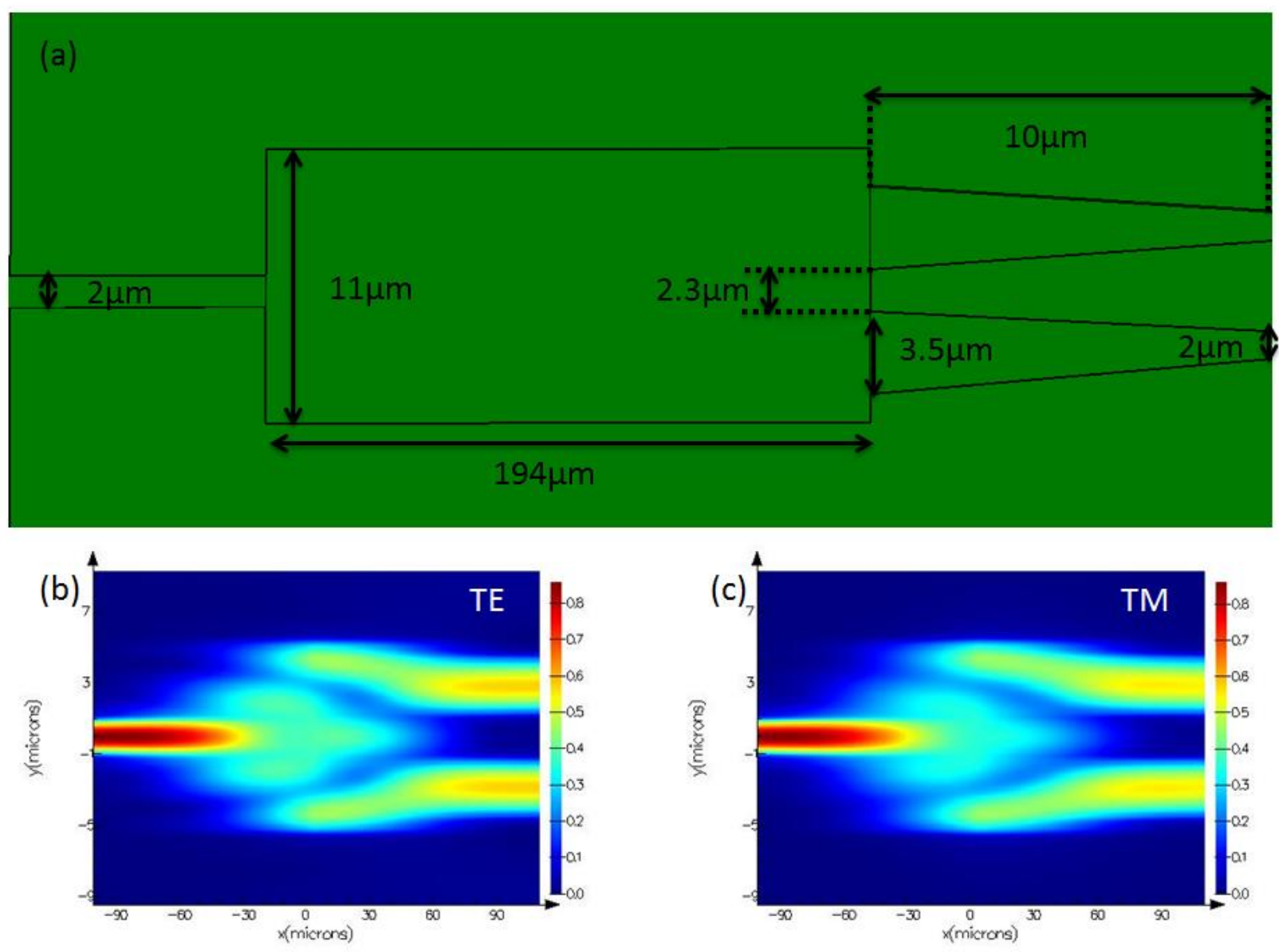

Figure 4. Polarization independent MMI design: (a) optimized geometry, (b) field intensity profile in TE, (b) field intensity profile in TM 


\section{CONCLUSION}

The influence of geometrical parameters on Ge-rich SiGe waveguides effective index, group index and dispersion birefringence has been evaluated. The proposed polarization insensitive geometry viability has been studied by calculating its possible tolerance to fabrication errors. The allowed margins are larger than the typical tolerances allowed in current CMOS foundries: variations of $+/-50 \mathrm{~nm}$ on the waveguide width, and $+/-10 \mathrm{~nm}$ on the etching depth allow keeping a polarization independence design. The study of the group index behavior shows that a different geometry has to be implemented if group index birefringence has to be minimized. Moreover, dispersion coefficient is positive and has a low dependency with the polarization and its absolute value is comparable to the dispersion coefficient obtained in SOI waveguides. Based on optimized waveguide geometry, a design of polarization insensitive 1x2 MMI is proposed for further realization of polarization insensitive Mach Zehnder interferometer. These results pave the way for Ge-rich $\mathrm{SiGe}$ polarization insensitive circuitry on bulk silicon substrate

\section{ACKNOWLEDGEMENTS}

The CARIPLO foundation is acknowledged for financial support through the grant EIDOS 2011-038. Marie Curie International Outgoing Fellowships through grant agreement PIOF-GA-2013-629292 MIDEX is partly acknowledged. This project has received funding from the European Research Council (ERC) under the European Union's Horizon 2020 research and innovation program (grant agreement $\mathrm{N}^{\circ} 639107$-INSPIRE) 


\section{REFERENCES}

[1] Camaco-Aguilera, R.E., Cai,Y., Patel, N.,Besette, J.T., Romagnoli, M., Kimerling, L.C., and Michel, J., “An electrically pumped germanium laser," Opt.Express 20(10),11316-11320(2012).

[2] Koerner, R., Oehme, M., Gollhofer, M., Schmid, M., Kostecki, K., Bechler, S., Widmann, D., Kasper, E., and Schulze, J., "Electrically pumped lasing from Ge Fabry-Perot resonators on Si," Opt.Express 23(11), 14815$14822(2015$

[3] Krishnamoorthy, A. V., Zheng, X., Feng, D., Lexau, J., Buckwalter, J. F., Thacker, H. D., Liu, F., Luo, Y., Chang, E., Amberg, P., Shubin, I., Djordjevic, S. S., Lee, J. H., Lin, S., Liang, H., Abed, A., Shafiiha, R., Raj, K., Ho, R., Asghari, M., and Cunningham, J. E., "A low-power, high-speed, 9-channel germanium-silicon electro-absorption modulator array integrated with digital CMOS driver and wavelength multiplexer," Opt. Express 22(10), 12289-12295 (2014).

[4] Chaisakul, P., Marris-Morini, D., Rouifed, M. S., Isella, G., Chrastina, D., Frigerio, J., Le Roux, X., Edmond, S., Coudevylle, J.-R., and Vivien, L., "23 GHz Ge/SiGe multiple quantum well electro-absorption modulator," Opt. Express 20(3), 3219-3224 (2012).

[5] Vivien, L., Polzer, A., Marris-Morini, D., Osmond, J., Hartmann, J. M., Crozat, P., Cassan, E., Kopp, C., Zimmermann, H. and Fédéli, J.M., "Zero-bias 40 Gbit/s germanium waveguide photodetector on silicon” Opt. Express 20, 1096-1101(2012).

[6] Chaisakul, P., Marris-Morini, D., Isella, G., Chrastina, D., Rouifed., M.-S., Le Roux, X., Edmond, S., Cassan, E., Coudevylle, J.-R., Vivien, L., “10-Gb/s Multiple Quantum-Well Waveguide Photodetector” IEEE Photon. Technol. Let. 23, 1430 (2011).

[7] Assefa, S., Xia, F., Bedell, S. W., Zhang, Y., Topuria, T., Rice, P.M. and Vlasov, Y.A., "CMOS-integrated high-speed MSM germanium waveguide photodetector," Opt.Express 18, 4986-4999 (2010).

[8] Virot, L., Crozat, P., Fédéli, J.-M., Hartmann, J.-M., Marris-Morini, D., Cassan, E., Boeuf, F., and Vivien, L., "Germanium avalanche receiver for low power interconnects," Nat. Commun. 5, 4957 (2014).

[9] Chaisakul, P., Marris-Morini, D., Frigerio, J., Chrastina, D., Rouifed, M. S., Cecchi, S., Crozat, P., Isella, G., and Vivien, L., "Integrated germanium optical interconnects on silicon substrates," Nat. Photonics 8(6), 482488 (2014).

[10] Vakarin,V., Chaisakul,P., Frigerio,J., Ballabio,A., Le Roux, X., Coudevylle,J.R, Bouville,D., Perez-Galacho,D., Vivien,L., Isella,G., and Marris-Morini,D., "Sharp bends and Mach-Zehnder interferometer based on Ge-richSiGe waveguides on SiGe graded buffer," Opt. Express 23(24), 30821-30826 (2015)

[11] Vivien, L., Laval, S., Dumont, B., Lardenois, S., Koster, A., Cassan, E., "Polarization-independent single-mode rib waveguides on silicon-on-insulator for telecommunication wavelengths, " Opt. Commun. 210, 43-49 (2002).

[12] Dulkeith, E., Xia, X., Schares, L., Green, W.M.J., Vlasov, Y.A., "Group index and group velocity dispersion in silicon-on-insulator photonic wires,” Opt. Express 14(9), 3853-3863 (2006). 